\title{
MULTIPLE FEATURE MODELS FOR IMAGE MATCHING
}

Juan Morales, Rafael Verdú, José Luis Sancho*

\author{
Universidad Politécnica de Cartagena \\ Information and Communication Technologies \\ Muralla del Mar, s/n, 30202 Cartagena, Spain \\ juan.morales@upct.es
}

\section{Luis Weruaga}

\author{
Austrian Academy of Sciences \\ Commission for Scientific Visualization \\ Donau-City Strasse 1, 1220 Vienna, Austria \\ weruaga@ieee.org
}

\begin{abstract}
The common approach to image matching is to detect spatial features present in both images and create a mapping that relates both images. The main drawback of this method takes place when more than one matching is likely. A first simplification to this ambiguity is to represent with a parametric model the point locus where the matching is highly likely, and then use a POCS (projection onto convex sets) procedure combined with Tikhonov regularization that results in the mapping vectors. However, if there is more than one model per pixel, the regularization and constraintforcing process faces a multiple-choice dilemma that has no easy solution. This work proposes a framework to overcome this drawback: the combined projection over multiple models based on the $L_{k}$ norm of the projection-point distance. This approach is tested on a stereo-pair that presents multiple choices of similar likelihood.
\end{abstract}

\section{INTRODUCTION}

Let $I(x, y)$ and $J(x, y)$ be two images, where $J(x, y)$ is assumed to be the result of the geometrical distortion of $I(x, y)$ by a continuous and differentiable spatial bijective mapping $\boldsymbol{\Psi}(x, y)=\left(\Psi_{x}(x, y), \Psi_{y}(x, y)\right)$, where $J(x, y)=I\left(\Psi_{x}(x, y), \Psi_{y}(x, y)\right)$. In this scenario, the goal is to find how both images, $I(x, y)$ and $J(x, y)$, are geometrically related, that is, the estimation of mapping $\boldsymbol{\Psi}(x, y)$. The spatial features of the image play an important role in that estimation, since an image with weak features would make this estimation ill-posed. An extreme case would be a flat image without contrast, in which there is no way to estimate whether there has been geometrical distortion at all.

A common methodology in this problem is to detect well-defined spatial features shared by both images and with the corresponding spatial shift to create the mapping [1]. From this idea, in previous works $[2,3,4]$ a method to estimate the mapping $\mathbf{\Psi}(x, y)$ for non-rigid motion was pre-

\footnotetext{
*This work is partially supported by the Spanish Ministerio de Ciencia y Tecnología, under grant TIC2002-03033.
}

sented. The proposed methodology consists of two separate and sequential steps:

1. Parametrization: From $I(x, y)$ and $J(x, y)$ to obtain a set of matching parametric models $\boldsymbol{\Omega}(\mathbf{x})$.

2. Regularization: To obtain the optimal mapping $\hat{\mathbf{\Psi}}_{o}$ from the parametric models $\boldsymbol{\Omega}(\mathrm{x})$.

These two steps could be recognized in Fig. 1, where the dotted line block encloses the first stage, and the lower block the second one. This classical approach has a main draw-

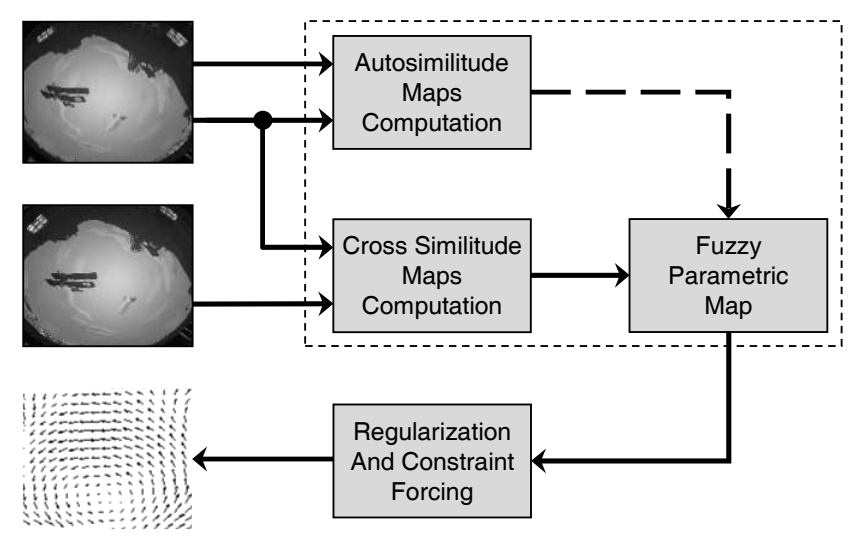

Fig. 1. Basic scheme to data correspondence

back, namely the detection of the common features is ambiguous when there is more than one likely matching. To alleviate this problem the use of dual models to represent the high similarity regions is proposed. The main issue of this work is the constraint-forcing procedure on second stage, because this is the point where model restrictions must be applied. More details about the motion estimation algorithm can be found in $[2,3,4]$.

The paper is organized as follows: section 2 presents the basic ideas to achieve a better image matching by using multiple models; section 3 presents comparative results to prove the validity of this approach and illustrate the estimation improvement; finally, section 4 contains the conclusions. 


\section{PROPOSED METHOD}

Let us assume that for each point $(x, y)$ we came up with a parametric model $\Omega(x, y)$ that represents explicitly or implicitly the point loci $\boldsymbol{\Phi}$ where the matching is highly likely. A parametric model will be present for the points $(x, y)$ where there is high evidence of feature correspondence between both images, and will be null for the remaining pixels.

\subsection{Parametric models}

For each pixel in $I(x, y)$ a Similarity Map (SM) $\rho_{\mathbf{c}}(x, y)$ of $(2 N+1)$-pixel width is computed according to $[2,3$, 4], which contains the similarity between a block of $I(x, y)$ centered at the pixel $\mathbf{c} \equiv\left(c_{x}, c_{y}\right)$ and a set of equal size blocks of $J(x, y)$ in a $(2 N+1)$ search length.

Two single parametrization models will be considered, and in addition in this work a dual model is also introduced to treat non-null disjoint regions capable of being parametrized independently. Fig. 2 shows some illustrative examples of various SMs, and the parametrization concept. In short, the regularization and constraint-forcing procedure needs to deal with following models:

1. Point model, $\boldsymbol{\Omega}_{p}=\left\{x_{0}, y_{0}\right\}$, computed from the center of mass of a cluster. This model will be applied in image isolated details.

2. Curve model, $\boldsymbol{\Omega}_{c}=\{\theta, a, b, c\}$, that is, a quadratic curve of coefficients $a, b$ and $c$ rotated by angle $\theta$. It mainly appears in object borders.

3. Dual model, $\boldsymbol{\Omega}_{m}=\left\{\boldsymbol{\Omega}_{1}, \boldsymbol{\Omega}_{2}\right\}$, where $\boldsymbol{\Omega}_{1}$ and $\boldsymbol{\Omega}_{2}$ could be any of the preceding models.

The constraint-forcing process consist of a model projection method. Specifically, the projection over multiple models is not an entirely solved issue, and will be treated below.
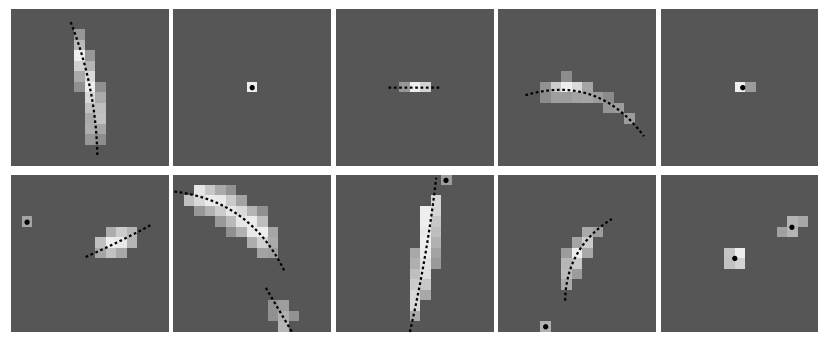

Fig. 2. Similarity map parametrization examples.

\subsection{Projection over multiple models}

If two models are present in $\boldsymbol{\Omega}$, that is to say, $\boldsymbol{\Omega}=$ $\left\{\boldsymbol{\Omega}_{1}, \boldsymbol{\Omega}_{2}\right\}$, the projection of a point or vector $\mathbf{x}_{0} \equiv\left(x_{0}, y_{0}\right)$ over this dual model must be averaged in some manner.
This projection need to be consistent with both models, and not to suppose a drastic rejection of none of the models. Moreover, it is intended that the projected point position, $\mathbf{x}_{0}^{\prime} \equiv\left(x_{0}^{\prime}, y_{0}^{\prime}\right)$, be so close to point $\mathbf{x}_{0}$ as both models restrictions allow, so that the variation of the spatial position will be minimum. In general, to attend all these requirements, it is possible to compute the point $\mathbf{x}_{0}^{\prime}$ in the way showed the following expression:

$$
\mathbf{x}_{0}^{\prime}=\mathbf{x}_{1}^{\prime} \frac{d_{2}}{d_{1}+d_{2}}+\mathbf{x}_{2}^{\prime} \frac{d_{1}}{d_{1}+d_{2}}
$$

where vectors $\mathbf{x}_{1}^{\prime}=\mathcal{P}\left\{\mathbf{x}_{0}, \boldsymbol{\Omega}_{1}\right\}$ and $\mathbf{x}_{2}^{\prime}=\mathcal{P}\left\{\mathbf{x}_{0}, \boldsymbol{\Omega}_{2}\right\}$ are the projections of the vector $\mathrm{x}_{0}$ over each of the models contained inside of the dual model, and $d_{1}$ and $d_{2}$ are respectively the Euclidean distances from such projections to the point $\mathbf{x}_{0}$. To compute $d_{1}$ and $d_{2}$ the rule $d_{i}=\left\|\mathbf{x}_{i}^{\prime}-\mathbf{x}_{0}\right\|$ will be applied.

\subsubsection{Averaged projection over point-point models}

The point-point dual models use to apear in image regions with clear and repetitive structures in the proximity of the analyzed pixel. In real images, the appearance probability of these models is substancial, in practice about $30 \%$ of dual models are of the type point-point.

Moreover, this is the most delicate situation, because the averaging process is not possible as such, and it is caused a unavoidable selection of one model. The election was resolved according to the point to project: the method selects the parameters of the model of minimal Euclidean distance to the point. This situation is shown in Fig. 3. Parameters

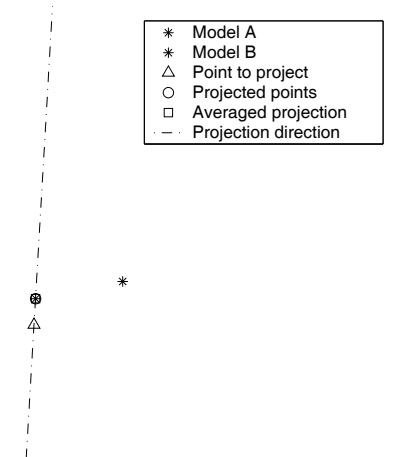

Fig. 3. Projection example over point-point model.

of both models, $\boldsymbol{\Omega}_{1}$ and $\boldsymbol{\Omega}_{1}$, will be $\boldsymbol{\Omega}_{1}=\left\{x_{1}, y_{1}\right\}$ and $\boldsymbol{\Omega}_{2}=\left\{x_{2}, y_{2}\right\}$. Although the projections over these models could be obtained in a direct manner, $\mathrm{x}_{1}^{\prime}=\mathrm{x}_{1}$ and $\mathrm{x}_{2}^{\prime}=\mathrm{x}_{2}$, due to special conditions of this type o dual models, and for correct application of expression (1), the coordinates of the projected vectors will be assigned according to (2).

$$
\mathbf{x}_{1}^{\prime} \equiv \mathbf{x}_{2}^{\prime}= \begin{cases}\mathbf{x}_{1} \text { if }\left\|\mathbf{x}_{1}-\mathbf{x}_{0}\right\|<\left\|\mathbf{x}_{2}-\mathbf{x}_{0}\right\| \\ \mathbf{x}_{2} \text { if }\left\|\mathbf{x}_{1}-\mathbf{x}_{0}\right\| \geqslant\left\|\mathbf{x}_{2}-\mathbf{x}_{0}\right\|\end{cases}
$$


where $\mathbf{x}_{2}=\left(x_{2}, y_{2}\right), \mathbf{x}_{1}=\left(x_{1}, y_{1}\right)$ and $\mathbf{x}_{0}=\left(x_{0}, y_{0}\right)$.

\subsubsection{Averaged projection over point-curve models}

Dual models of the type point-curve used to appear in similar situations to the previous section, but not for so welldefined structures. It may be considered a transition situation between the point-point and curve-curve dual models. For this reason and depending on the circumstances it is possible that these regions were detected in several ways. This dual model is the situation of least appearance probability. According to the experiments with real images, this probability could be summarized about a $20 \%$ of dual models. To obtain the averaged projection, a direction of projection must be determined: the direction that join the point to project and the point representing the model will be taken. So that, the projection over the point model comes from the own coordinates that defines the point model, whereas the projection on the curve model could be obtained from the intersection of the projection direction and the curve model. Once the projections on both models have been computed, the final projected vector $\mathbf{x}_{0}^{\prime}$ will be determined in a weighted averaging process by means of expression (1). Fig. 4 illustrates the projection process. Assuming that the

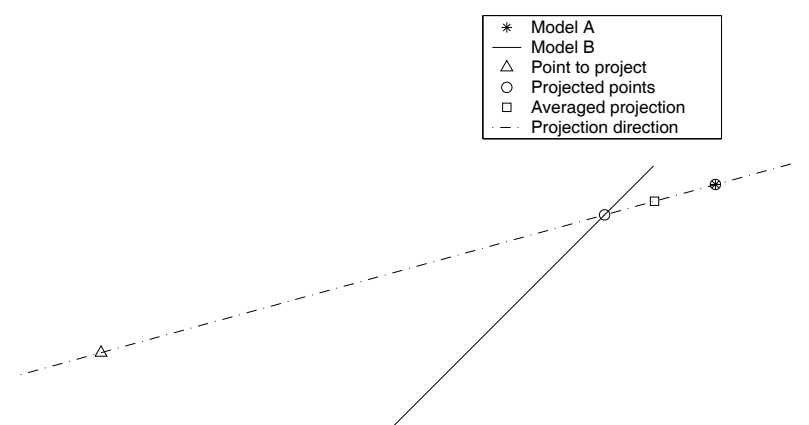

Fig. 4. Projection example over point-curve model.

first model is the point model, the model parameters will be $\boldsymbol{\Omega}_{1}=\left\{x_{1}, y_{1}\right\}$ and $\boldsymbol{\Omega}_{2}=\{\theta, a, b, c\}$. According to the previous ideas, the projection over the point model is obtained in a direct way, $\mathbf{x}_{1}^{\prime}=\mathcal{P}\left\{\mathbf{x}_{0}, \Omega_{1}\right\}=\mathbf{x}_{1}$, whereas the projection over the curve model, $\mathbf{x}_{2}^{\prime}$, is computed by means of the following successive expressions, that is, (3), (4), (5) and (6).

$$
\begin{aligned}
& \varphi=\arctan \left(\frac{y_{1}-y_{0}}{x_{1}-x_{0}}\right) \quad \Longrightarrow \quad m=\tan (\varphi-\theta) \\
& {\left[\begin{array}{l}
\hat{x}_{0} \\
\hat{y}_{0}
\end{array}\right]=\left[\begin{array}{rr}
\cos (\theta) & \sin (\theta) \\
-\sin (\theta) & \cos (\theta)
\end{array}\right]\left[\begin{array}{l}
x_{0} \\
y_{0}
\end{array}\right]} \\
& \left.\begin{array}{l}
\hat{y}=m\left(\hat{x}-\hat{x}_{0}\right)+\hat{y}_{0} \\
\hat{y}=a \hat{x}^{2}+b \hat{x}+c
\end{array}\right\} \Longrightarrow \\
& \left\{\begin{array}{l}
\hat{x}=\frac{-(b-m) \pm \sqrt{(b-m)^{2}-4 a\left(c+m \hat{x}_{0}-\hat{y}_{0}\right)}}{2 a} \\
\hat{y}=a \hat{x}^{2}+b \hat{x}+c
\end{array}\right.
\end{aligned}
$$

$$
\left[\begin{array}{l}
x_{2}^{\prime} \\
y_{2}^{\prime}
\end{array}\right]=\left[\begin{array}{rr}
\cos (\theta) & -\sin (\theta) \\
\sin (\theta) & \cos (\theta)
\end{array}\right]\left[\begin{array}{l}
\hat{x} \\
\hat{y}
\end{array}\right]
$$

If $a=0$, it is not possible to apply equations in (5) due to an indetermination. In that case, the process can still be applied by replacing (5) by (7).

$$
\begin{aligned}
& \left.\begin{array}{l}
\hat{y}=m\left(\hat{x}-\hat{x}_{0}\right)+\hat{y}_{0} \\
\hat{y}=b \hat{x}+c
\end{array}\right\} \Longrightarrow \\
& \left\{\begin{array}{l}
\hat{x}=\frac{-\left(c+m \hat{x}_{0}-\hat{y}_{0}\right)}{b-m} \\
\hat{y}=b \hat{x}+c
\end{array}\right.
\end{aligned}
$$

\subsubsection{Averaged projection over curve-curve models}

Curve-curve dual models are the most usual models, and also the produce the most ambiguous situation, because more freedom over the projected point location is allowed. About a $50 \%$ of dual models are of this type. Fig. 5 shows the procedure of averaged projection for the case. The

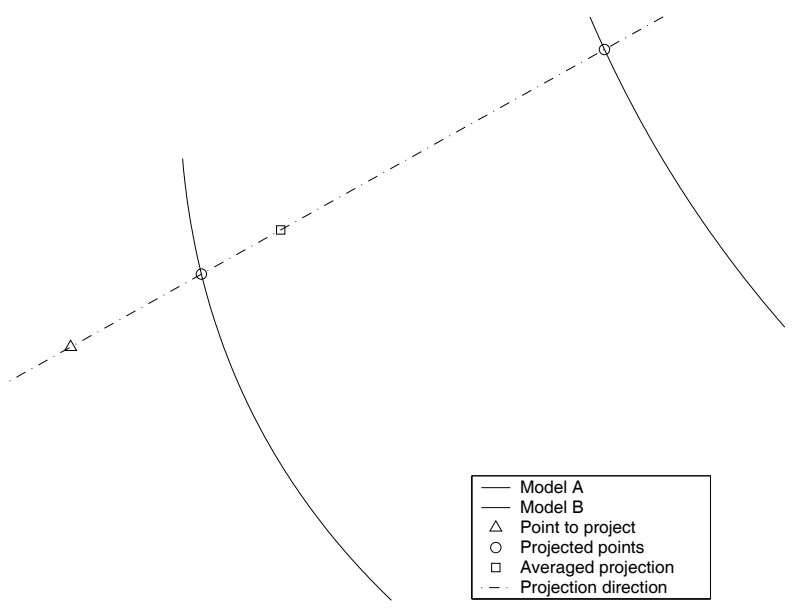

Fig. 5. Projection example over curve-curve model.

model parameters, $\boldsymbol{\Omega}_{1}$ and $\boldsymbol{\Omega}_{2}$ will be $\boldsymbol{\Omega}_{1}=\left\{\theta_{1}, a_{1}, b_{1}, c_{1}\right\}$ and $\boldsymbol{\Omega}_{2}=\left\{\theta_{2}, a_{2}, b_{2}, c_{2}\right\}$. The first step to achieve the dual projection is to determine, like it is showed in (8), the normal direction to the mean angle of the orientations of both parametric models, $\varphi$. From this value of $\varphi$ and for each model orientation, $\theta_{1}$ and $\theta_{2}$, the slope of a new $\theta$-derotated line, $m_{1}$ and $m_{2}$ respectively, is computed as follows:

$$
\begin{aligned}
& \varphi=\left\{\begin{array}{cc}
\frac{\theta_{1}+\theta_{2}+\pi}{2} & \text { if } \quad\left|\theta_{1}-\theta_{2}\right|<\pi \\
\frac{\theta_{1}+\theta_{2}}{2} & \text { if } \quad\left|\theta_{1}-\theta_{2}\right| \geq \pi
\end{array}\right. \\
& \Longrightarrow\left\{\begin{array}{l}
m_{1}=\tan \left(\varphi-\theta_{1}\right) \\
m_{2}=\tan \left(\varphi-\theta_{2}\right)
\end{array}\right.
\end{aligned}
$$

In this point, the intersections of each curve, defined by its model parameters, and a line of direction $\varphi$ are computed. 
In this way, expressions (4), (5) or (7), and (6) can be applied once per model and with the corresponding values of $\theta_{i}, a_{i}, b_{i}, c_{i}$ and $m_{i}$ parameters. Finally, from the computed projections, $\mathbf{x}_{1}^{\prime}$ and $\mathbf{x}_{2}^{\prime}$, expression (1) must be applied to determinate the averaged projection point, $\mathbf{x}_{0}^{\prime}$.

It must be noted that when the original point position, $\mathbf{x}_{0}$, is outside the region located between both models, the projected point $\mathbf{x}_{0}^{\prime}$ will be inside it, in an initial approaching operation. Once this situation is reached, the use of (1) iteratively tends to take no effect over the projected vector $\mathbf{x}_{0}^{\prime}$. This fact is not a drawback of the algorithm, but it is the fundamental goal of the method. In this manner, no hard decisions are taken and the neighboring pixels influence (single models and regularization procedure) pull the final projected vector towards the correct model, always inside the region described by the dual model.

\section{RESULTS}

Fig. 6 shows comparative results of image matching for a pair of $500 \times 500$ sample images. Both initial images, Fig. 6(a) and Fig. 6(b), are taken from a semispheral dome, so the interimage deformation proceeds from different camera point of view and the own dome curvature. Fig. 6(c) and Fig. 6(d) show the results of best matching reached from a single model estimation. Some errors in pixel matching can be clearly observed. When dual models are considered, Fig. 6(e) and Fig. 6(f), these errors are avoided and no visual difference can be detected between Fig. 6(e) and Fig. 6(a). Anyhow, note that deformation estimation results poor, although better, in regions around image corners. In these points, different objects or features are present in each image, therefore perfect matching is not possible.

\section{CONCLUSIONS}

In this work, the use of simple dual parametrization models to deal with ambiguous matching situations is proposed. This cases appear when characteristics of the image induces ambiguity in terms of multiple likely choice. The showed results prove the efficiency of this approach. Most of pixel assignment errors can be solved with this idea. The proposed method alleviates the problem that presents some classic matching algorithms. Typical block-matching algorithms suffer from this limitation. The dual parametrization method is been applied today to registration of a wide kind of images with excellent results. The extension of the method to more complex models is under test at present.

\section{REFERENCES}

[1] A. Goshtasby, et al., "A region-based approach to digital image registration with subpixel accuracy," IEEE

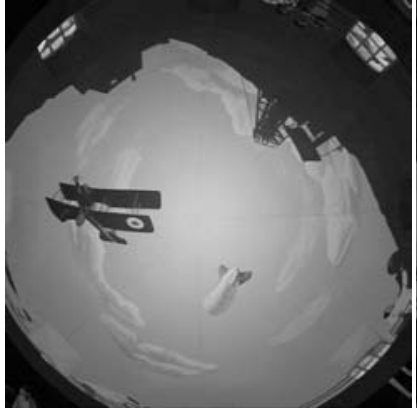

(a) Image 1 (reference image)

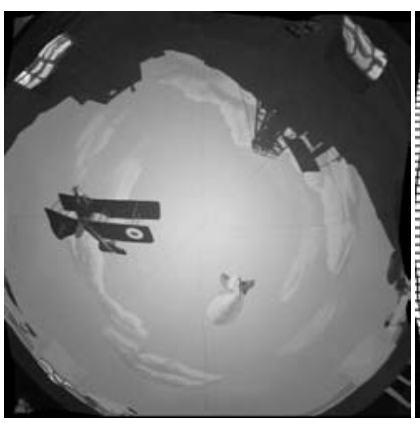

(c) Unwarped image (single models) (d) Unwarping grid (single models)

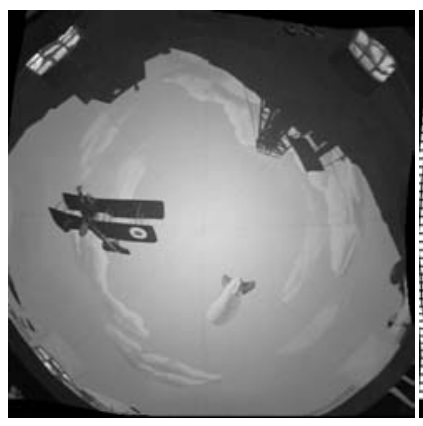

(e) Unwarped image (dual models)

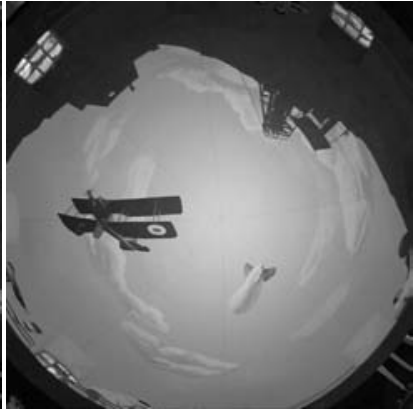

(b) Image 2 (target image)
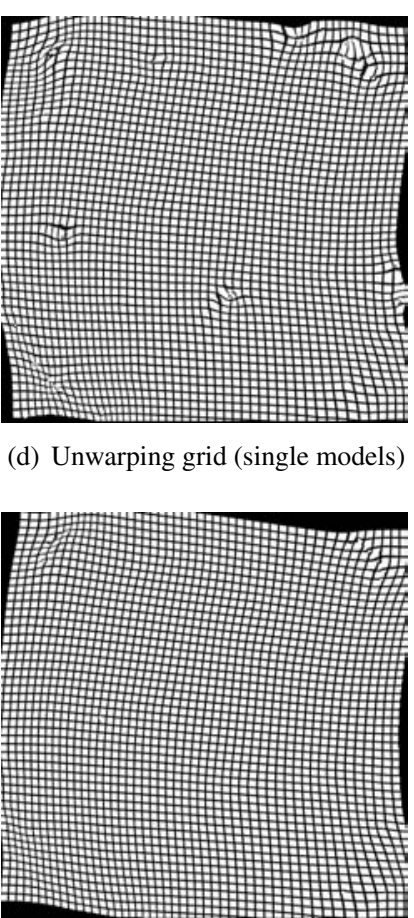

(f) Unwarping grid (dual models)
Fig. 6. Matching estimation results on sample images.

Trans. GRS, vol. 24, pp. 390-399, 1986.

[2] L. Weruaga, et al., "Estimating volumetric motion in human thorax with parametric matching constraints," IEEE Trans. MI, vol. 22, pp. 766-772, June 2003.

[3] J. Morales, et al., "Fuzzy non-rigid motion estimation robust to rotation," in Proc. IEEE ICIP'04, Singapore, 2004, pp. 1489-1492.

[4] J. Morales, et al., "Non-rigid motion estimation based on fuzzy models," in Proc. IEEE ICDSP'02, Santorini, Greece, 2002, pp. 559-562. 